\title{
Step-Ladder Expansive Cranioplasty after Decompressive Craniotomy and Expansive Duraplasty: Discussing Possibilities on a Mathematical Model
}

\author{
S. K. Sengupta ${ }^{1}$ \\ ${ }^{1}$ Department of Neurosurgery (Surgical Ward III), Base Hospital Delhi \\ Cantonment, New Delhi, India \\ Indian J Neurosurg 2015;4:15-21.
}

Address for correspondence Col (Dr.) S. K. Sengupta, MS, MCh, Department of Neurosurgery (Surgical Ward III), Base Hospital Delhi Cantonment, New Delhi 110010, India

(e-mail: sudipkumarsengupta14@rediffmail.com).

\begin{abstract}
Keywords

- decompressive craniotomy

- step-ladder expansive cranioplasty

- mathematical model

Background Role of decompressive craniectomy in reducing intracranial pressure is well established. However, it comes with a cost of requiring a second surgery in the form of cranioplasty without which unacceptable hemodynamic consequences occur. It is generally felt that a credible alternative is required.

Objective The aim of the study is to devise a mathematical model, which closely represents the cranium and intracranial contents, on which various alternatives can be evaluated with reproducible results, and to work out the effects of a novel technique of expansive cranioplasty on that model.

Methods A mathematical model was designed based on the presumption that dura forms a watertight bag-containing brain, floating in cerebrospinal fluid (CSF). A model for an expansive cranioplasty was designed, and its ability to provide the space required to allow volume expansion and to achieve adequate reduction in intracranial pressure (ICP) was evaluated on this model.

Results The mathematical model could closely reproduce the surface area-volume relationships in the published literature. Based on the calculations on the model, it was found that a projection of dural outpouching of $0.83 \mathrm{~cm}$ beyond the craniectomy margin on either side of a bilateral $12 \times 15 \mathrm{~cm}$ elliptical craniectomy defect is required to achieve and accommodate a volume expansion of $157 \mathrm{~cm}^{3}$, which was recorded to be the maximum volume expansion in the reviewed literature. A two-step step-ladder cranioplasty can be constructed to achieve an increase in cranial width by 1.1 to $1.3 \mathrm{~cm}$ on each side.

Conclusion Calculations based on the present model indicate that a two-step expansive cranioplasty can accommodate adequate volume expansion while alleviating the ill effects of a craniectomy and necessity of a second surgery. However, these are discussions on mathematical model, based on multitude of assumptions and approximations, and hence these discussions require clinical trials to validate the findings.
\end{abstract}

(c) 2015 Neurological Surgeons' Society of India 


\section{Introduction}

Raised intracranial pressure (ICP) and the resultant decrease in cerebral perfusion pressure is the root cause of deterioration in various clinical settings of brain injury, which include those inflicted by traumatic and ischemic insults. Role of decompressive craniectomy in reducing ICP is well established. ${ }^{1-3}$ However, it often requires a second surgery in the form of cranioplasty. Bone removal is known to give rise to complications in the form hydrocephalus and "syndrome of trephined." ${ }^{4}$ There is a felt necessity to look for other alternatives.

A mathematical model was designed, based on the laws of physics and solid geometry, to simulate the intracranial compartment and discuss the various effects of volume and pressure changes occurring in the event of craniectomy performed in the face of a raised ICP. Some of the observations of existing studies, which have been accepted universally and incorporated in neurosurgical practice, were studied on this model to derive certain relevant data, which are not available at present but are required to formulate new treatment strategies.

The volume expansion required to achieve adequate pressure reduction was noted from the existing widely accepted studies ${ }^{5,6}$ and a novel design of expansive craniectomy was assessed for its ability to accommodate the volume, thereby allowing a possibility of doing away with the second surgery for replacing the bone flap and avoiding the complications of sunken flap syndrome.

\section{Methods}

\section{The Mathematical Model to Represent the Intracranial Compartment}

A mathematical model was designed, based on the presumption that dura forms a watertight bag-containing brain floating in CSF, which is again reconstituted once a dural closure has been achieved. For this study, the dural bag has been presumed to be hemispherical in shape, with the flat circular surface of the hemisphere lying over the base of the skull ( - Fig. 1A). Volume of a hemisphere is $2 / 3 \pi R^{3}$, where $R$ is the radius of the sphere, of which the hemisphere has been carved out. Considering the intracranial volume to be approximately of $1,500 \mathrm{~mL},{ }^{7,8} R$ would work out to be $8.945 \mathrm{~cm}$ (a value of $9 \mathrm{~cm}$ has been used for the subsequent calculations).

On the basis of Pascal law, it can be stated that the ICP is exerted equally on the dural bag tangentially all over its wall, always trying to expand it outward (-Fig. 1A). Any expansion in the size of the dural bag is, however, prevented by the intact rigid cranium around it. A craniectomy defect can be circular, rectangular, or oval in shape. The defect with a given surface area, of any shape, has been represented in the model by a circular area of equal surface area, having a radius $r_{1}(-$ Fig. 1B). The maximum distance of the imaginary horizontal line drawn at the level of the craniectomy defect to the normal location of the dura in unoperated skull, obtained by drawing a perpendicular from the center of the imaginary sphere representing the

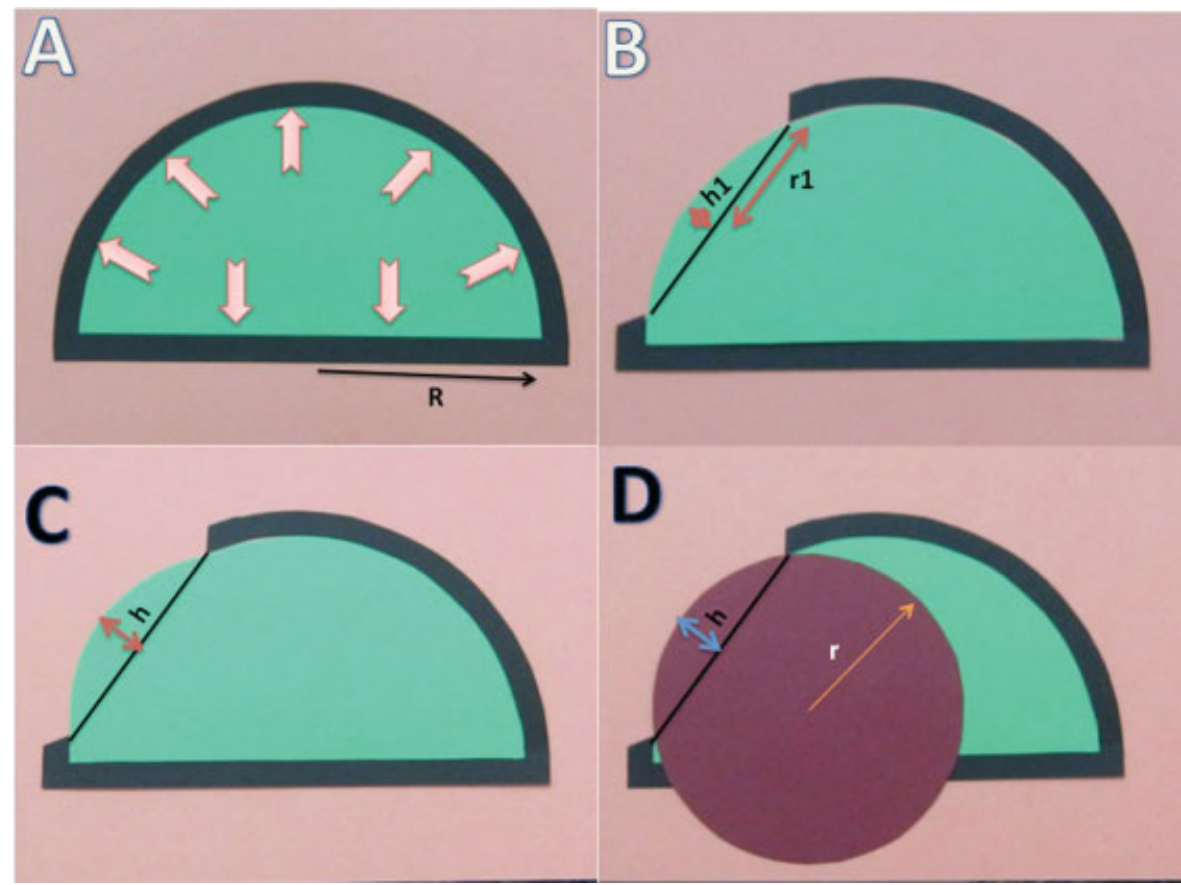

Fig. 1 (A) Black border represents the skull and green area represents the hemispherical intracranial dural bag with radius $R$. Pink arrows indicate the direction of forces acting outward on the dural bag. (B) Craniectomy defect of radius $r_{1}$, maximum distance of the inner table from an imaginary line drawn at the level of the craniectomy defect being $h_{1}$. (C) After volume augmentation of the dural sac at the craniectomy site the maximum projection of the dural sac beyond the craniectomy margin $h$. (D) The hemispheric projection of the dural sac beyond the craniectomy margin can be considered be a section of another imaginary sphere of diameter $r$. 


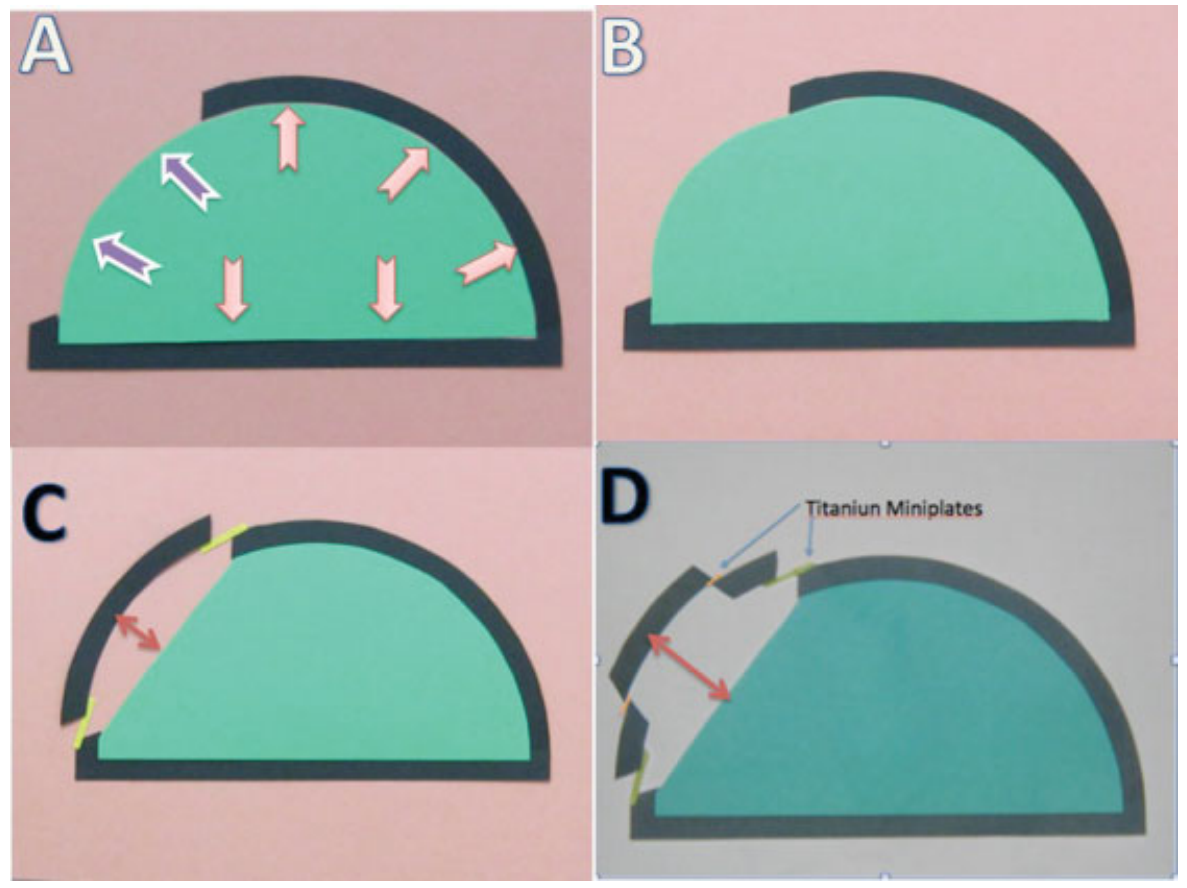

Fig. 2 (A) Black border represents the skull and green area represents the hemispherical intracranial dural bag. At the craniectomy site, the dura is exposed to the intracranial pressure, purple arrows indicating the vector of forces that can work on the dura effectively stretching it. (B) Volume expansion of the dural bag at the craniectomy site. (C) A single-step step-ladder expansive cranioplasty constructed by fixing the free bone flap and the cranium on the two opposite surfaces of a titanium miniplate. The double-headed arrow indicates the distance from the center of the craniectomy defect to the pole of the inner table of the cranioplasty construct. (D) A double-step step-ladder expansive cranioplasty.

craniectomy defect to the pole, is represented in the model by $h_{1}$.

$$
\left.h_{1}=\mathrm{R}-\sqrt{(} R^{2}-r_{1}^{2}\right)
$$

The volume of the part of the dural sac $(E)$, without any stretching, calculated from the edge of the defect is

$$
2 / 3 \pi r_{1}{ }^{2} h_{1}
$$

The outpouching of the dural bag, after a dural closure has been achieved (-Fig. 1C), has been considered to be represented by a section of an imaginary sphere with a radius $r$ (-Fig. 1D). The maximum distance of the dural outpouching from the craniectomy margin is represented by $h$.

Whenever a craniectomy is done, the vector of forces become free to stretch the dural bag over the portion of the craniectomy defect ( $\mathbf{- F i g . 2}$ ). Because the pressure exerted at each point is equal, this is expected to cause a spherical outpouching. It is the volume of this outpouching that will determine the volume expansion achieved and will require to be accommodated by an expansive craniectomy technique.

For a circular craniectomy defect ( - Fig. 1) of known size $\left(r_{1}\right)$ and volume $(V)$, maximum distance of the sac from the craniectomy margin $(h)$ can be calculated by the formula

$$
h=3 V / 2 \pi r_{1}^{2}
$$

The projection of the dural bag beyond the preoperative dural limit

$$
(H)=h-h_{1}
$$

The relationship of $r_{1}$ to the $r$ (the radius of the sphere of which this outpouching is a part) is given by the formula

$$
r=\left(r_{1}{ }^{2}+h_{1}{ }^{2}\right) / 2 h_{1}
$$

\section{Designing Expansive Cranioplasty}

A craniectomy procedure aims to increase the available intracranial volume, thereby reducing the ICP. Literature review was done to find out the median volume expansion achieved after craniectomy in series with satisfactory ICP reduction and maximum volume expansion achieved among all the studies in which volume expansion has been documented after craniectomy procedures. To accommodate this additional volume, the projection of the dural bag required to take place, beyond the preoperative state, was calculated for different craniotomy size. A step-ladder pattern cranioplasty technique, in which the free bone flap and the craniectomy edge are fixed on two opposite surfaces of titanium miniplates (-Fig. 3), was evaluated for its applicability.

\section{Results}

Considering the volume of the cranial contents to be 1,500 $\mathrm{cm}^{3}$, it can be represented by a hemisphere of $9 \mathrm{~cm}$ radius. 
A

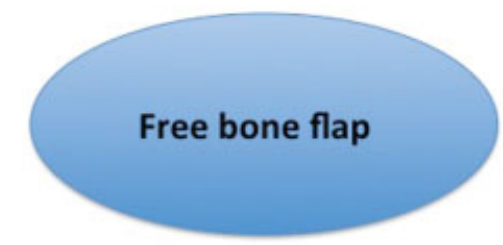

C

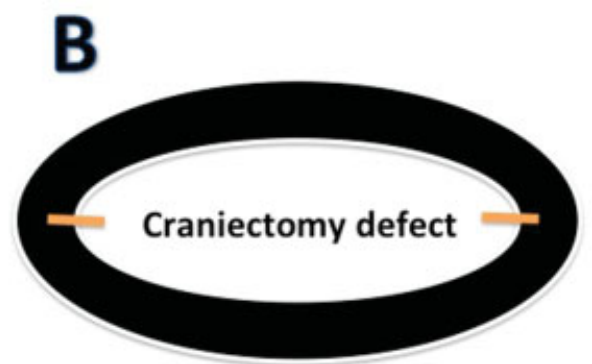

D

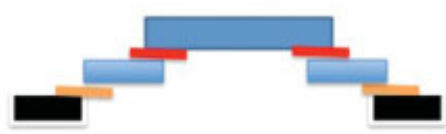

Sagittal view

Step ladder cranioplasty

Fig. 3 Step-ladder expansive cranioplasty. (A) Free bone flap. (B) Black line representing the craniectomy margin with the white central portion representing the craniectomy defect. Titanium miniplates, represented by the yellow lines, are fixed on the outer surface of the cranium with screws. (C) A central portion of the free bone flap has been removed leaving a ring of $1.5 \mathrm{~cm}$ width. Miniplates fixed to the outer surface of the cranium are fixed to the inner surface of the bony ring. A second set of titanium miniplates, represented by red lines, are fixed to the outer surface of the bony ring on one end and to the inner surface of the bony inner disc on the other end. Arrows indicating the directions to fix the miniplates. (D) Sagittal section of the construct.

The results have been summarized in $\boldsymbol{\bullet}$ Table 1.

1. Conversion of elliptical craniectomy defects of different surface area in the published studies to equivalent circular defects in the present model.

(a) An elliptical craniectomy defect of $12 \times 15 \mathrm{~cm}$ has a surface area of $141.42 \mathrm{~cm}^{2}$; an equivalent circular defect of equal surface area will have a diameter of $13.4 \mathrm{~cm}$.

(b) An elliptical craniectomy defect of $12 \times 08 \mathrm{~cm}$ has a surface area of $75.42 \mathrm{~cm}^{2}$; an equivalent circular defect of equal surface area will have a diameter of $9.79 \mathrm{~cm}$.

(c) A craniectomy defect of $67.9 \mathrm{~cm}^{2}$ will be represented by a circular defect of $9.29 \mathrm{~cm}$ diameter.

2. Measurements of dural sac at the craniectomy site.

(a) Maximum distance from the line joining the craniectomy margins to the outer margin of unexpanded dura $\left(h_{1}\right)$ :

- For a $13.4-\mathrm{cm}$ diameter circular (equivalent to $12 \times 15 \mathrm{~cm}$ elliptical) craniectomy defect: $3.00 \mathrm{~cm}$.

- For a $9.79-\mathrm{cm}$ diameter circular (equivalent to $12 \times 08 \mathrm{~cm}$ elliptical) craniectomy defect: $1.45 \mathrm{~cm}$.

- For a $67.9-\mathrm{cm}^{2}$ (9.29-cm diameter circular) craniectomy defect: $1.3 \mathrm{~cm}$.

(b) For an additional volume of $124 \mathrm{~cm}^{3}$, to be accommodated, the required increase in the height of the dural pouch projecting from craniectomy defects $\left(h-h_{1}\right)$ :
- For a 13.4-cm diameter circular (equivalent to $12 \times 15 \mathrm{~cm}$ elliptical) craniectomy defect: $1.32 \mathrm{~cm}$.

- For a 9.79-cm diameter circular (equivalent to $12 \times 08 \mathrm{~cm}$ elliptical) craniectomy defect: $2.47 \mathrm{~cm}$.

- For a $67.9-\mathrm{cm}^{2}$ (9.29 $\mathrm{cm}$ diameter circular) craniectomy defect: $2.74 \mathrm{~cm}$

(c) For an additional volume of $157.6 \mathrm{~cm}^{3}$, to be accommodated, the required increase in the height of the dural pouch projecting from craniectomy defects $\left(h-h_{1}\right)$ :

- For a 13.4-cm diameter circular (equivalent to $12 \times 15 \mathrm{~cm}$ elliptical) craniectomy defect: $1.67 \mathrm{~cm}$.

(d) For an additional volume of $157.6 \mathrm{~cm}^{3}$, to be accommodated in a bilateral hemicraniectomy, in which each side has to accommodate a volume expansion of $78.8 \mathrm{~cm}^{3}$, the required increase in the height of the dural pouch projecting from craniectomy defects $\left(h-h_{1}\right)$ :

- For a 13.4-cm diameter circular (equivalent to $12 \times 15 \mathrm{~cm}$ elliptical) craniectomy defect: $0.83 \mathrm{~cm}$.

(e) For an additional volume of $124 \mathrm{~cm}^{3}$, to be accommodated in a bilateral hemicraniectomy, in which each side has to accommodate a volume expansion of $62 \mathrm{~cm}^{3}$, the required increase in the height of the dural pouch projecting from craniectomy defects $\left(h-h_{1}\right)$ :

- For a $9.79-\mathrm{cm}$ diameter circular (equivalent to $12 \times 8 \mathrm{~cm}$ elliptical) craniectomy defect: $1.23 \mathrm{~cm}$ 

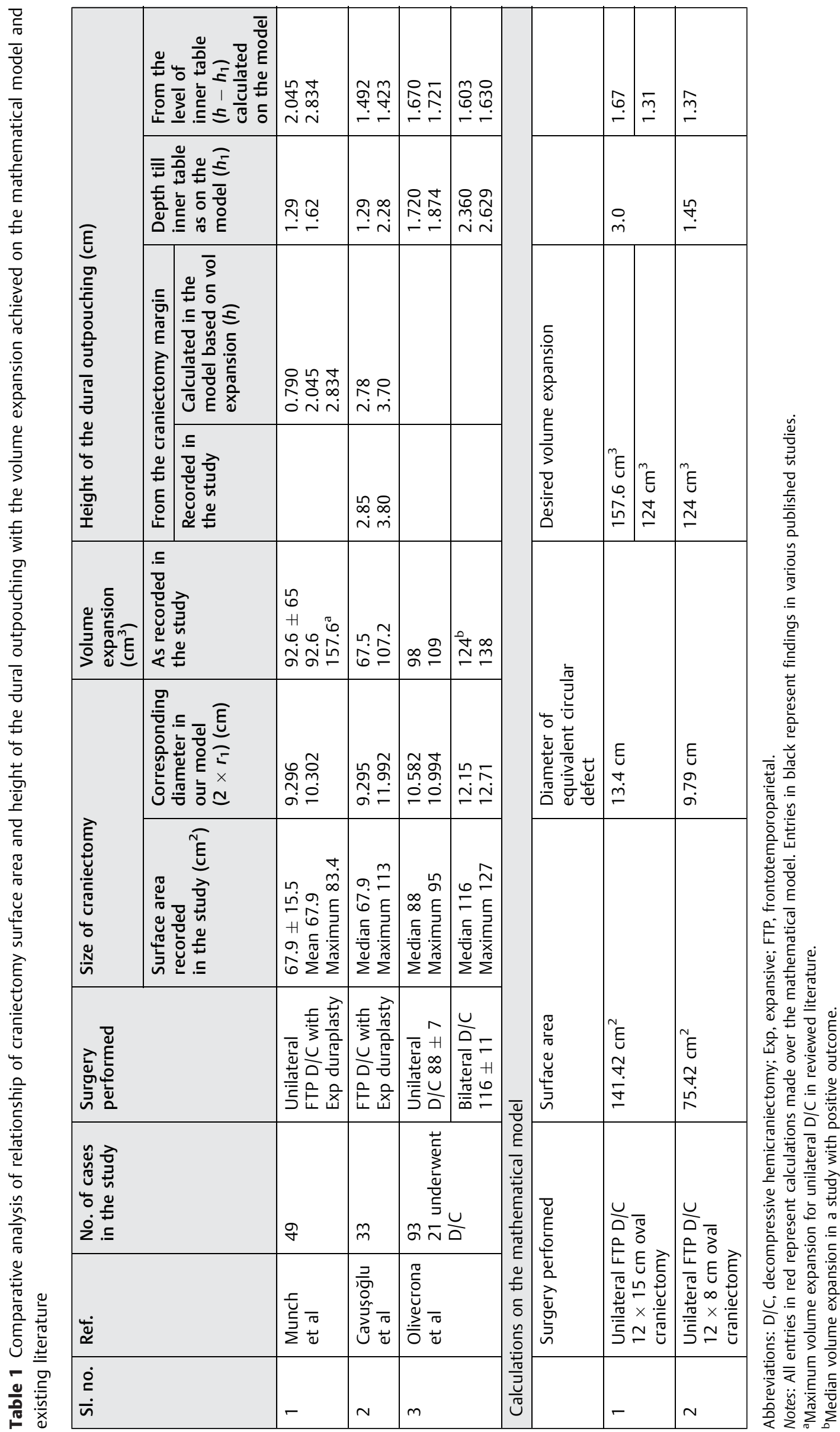
- For a $67.9-\mathrm{cm}^{2}(9.29 \mathrm{~cm}$ diameter circular $)$ craniectomy defect: $1.37 \mathrm{~cm}$.

(f) If an expansive cranioplasty is constructed, widening the craniectomy site by $1 \mathrm{~cm}$, it can allow a volume expansion of:

- For a 13.4-cm diameter circular (equivalent to $12 \times 15 \mathrm{~cm}$ elliptical) craniectomy defect: $94 \mathrm{~cm}^{3}$.

- For a 9.79-cm diameter circular (equivalent to $12 \times 8 \mathrm{~cm}$ elliptical) craniectomy defect: $50 \mathrm{~cm}^{3}$.

\section{Discussion}

Raised ICP and the resultant decrease in cerebral perfusion pressure is the root cause of deterioration in various clinical settings of brain injury. The fact that decompressive craniectomy effectively reduces ICP is well established. ${ }^{1-3,9}$

Some of the problems that crop up after craniectomy, which offset the obvious advantages of the procedure, thereby annulling the benefits accrued by opening the cranium and reduction in ICP, are postcraniectomy cerebral edema, ${ }^{10,11}$ derangements of cerebral autoregulation, infarct of the prolapsed brain parenchyma, intracerebral hemorrhages, ${ }^{12}$ and sunken flap syndrome.

Efforts have been made to alleviate some of these problems by resorting to various alternative, novel surgical techniques, ${ }^{13-15}$ and modifications ${ }^{16,17}$ with varied amount of success; however, neither have they gained universal acceptance nor have they been able to take care of all the drawbacks. A wide durotomy and expansive duraplasty have been advocated to accommodate the surplus brain volume caused by the postoperative edema ${ }^{18}$ in view of the perceived inevitability of the postcraniectomy cerebral edema.

The postcraniectomy complications can be broadly divided into two groups. First set of complications are due to bone flap removal, namely, sunken flap syndrome and postcraniectomy hydrocephalus. The second set of complications are secondary to the hemodynamic changes brought about by dural opening, namely, postoperative cerebral edema, kinking of cerebral veins, and infarct. First set of complications could be avoided if it was possible to develop a technique of expansive cranioplasty, creating required additional space to accommodate desired volume expansion, while replacing the bone flap on completion of surgery.

Experimenting on these concepts in clinical settings, while established alternatives exist, is unethical at the best. Therefore, a mathematical model was created to juggle with the available information and finally a cranioplasty design was created on this model that would allow adequate volume expansion.

Three studies were found in the literature that compared the craniectomy size with the volume expansion achieved. ${ }^{5,6,18}$ Although 82 cases of unilateral decompressive craniectomy were reported by Munch et al and Cavuşoğlu et al, 22 operated cases reported by Olivecrona et al underwent either unilateral or bilateral craniectomy based on their computed tomography (CT) findings. The maximum craniectomy size reported for an unilateral surgery was $113 \mathrm{~cm}^{2}$. Largest among the three studies was by Munch et al with 49 cases with a mean craniectomy size of $67.9 \mathrm{~cm}^{2}$ and a mean volume expansion of $92.6 \mathrm{~cm}^{3}$. In their study, the maximum craniectomy size was recorded to be $83.4 \mathrm{~cm}^{2}$ and the maximum volume expansion achieved was $157.6 \mathrm{~cm}^{3}$. The study by Olivecrona et al showed encouraging results with decompressive hemicraniectomy for cases of severe traumatic head injury with resistant raised ICP, a maximum volume expansion of $127 \mathrm{~cm}^{3}$, and an ICP reduction by $41 \%$.

Calculations on the mathematical model showed that a volume expansion of $46.1,67.5$, and $107.2 \mathrm{~cm}^{3}$ achieved over craniectomy defects of $51.5,67.9$, and $113 \mathrm{~cm}^{2}$, respectively, will require the dural outpouchings to project $2.3,2.78$, and $3.70 \mathrm{~cm}$ beyond the craniectomy margin. These measurements were similar to the findings recorded in the study by Cavuşoğlu et al. ${ }^{5}$

Mathematical calculations on the model showed that a projection of dural outpouching of $1.32 \mathrm{~cm}$ beyond the craniectomy margin of a unilateral $12 \times 15 \mathrm{~cm}$ elliptical craniectomy defect is required to achieve and accommodate a volume expansion of $124 \mathrm{~cm}^{3}$, which was recorded to be the mean volume expansion in the study published by Olivecrona et al, reporting a positive outcome of the study. A projection of dural outpouching of $0.83 \mathrm{~cm}$ beyond the craniectomy margin on either side of a bilateral $12 \times 15 \mathrm{~cm}$ elliptical craniectomy defect is required to achieve and accommodate a volume expansion of $157 \mathrm{~cm}^{3}$, which was recorded to be the maximum volume expansion in the study published by Munch et al and is by far the maximum volume expansion recorded in any study after a unilateral decompressive hemicraniectomy performed by the standard technique. Considering the thickness of the bones at the anterior and posterior margins of a craniectomy defect to be 5 to $6 \mathrm{~mm}$ and the width of the miniplates $0.5 \mathrm{~mm}$, a step-ladder cranioplasty can be constructed to achieve an increase in cranial width by 1.1 to $1.3 \mathrm{~cm}$ on each side. The width can be increased further by drilling out a circular area of inner table and dipole from the center of the bone flap by 2 to $3 \mathrm{~mm}$. Performing a three-step step-ladder cranioplasty or leaving a craniectomy defect of $3 \mathrm{~cm}$ diameter at the center of the bone flap can also be considered as alternatives. A two-step stepladder cranioplasty with a gain of $1.0 \mathrm{~cm}$ width can, in a bilateral $12 \mathrm{~cm} \times 15 \mathrm{~cm}$ elliptical craniotomy and expansive cranioplasty, accommodate $184 \mathrm{~cm}^{3}$ of additional volume, and can effectively allow more ICP reduction by taking the inward pressure of the tensile strength of the scalp flap off the dural pouch.

\section{Conclusion}

Calculations based on the present model indicate that a twostep step-ladder expansive cranioplasty can provide ample space to accommodate the extra volume created by an expansive duraplasty, if the craniectomy is large $(12 \times 15 \mathrm{~cm})$ and performed bilaterally. 
However, it is just a mathematical model, based on multitude of assumptions and approximations, and hence cannot be taken on the face value. If the logics forwarded, after critical review by the neuroscientists, gain some acceptance, various components of the study can be tested in animal models and in suitable, very selective clinical settings.

\section{Disclosure}

None to declare.

\section{References}

1 Jiang JY, Xu W, Li WP, et al. Efficacy of standard trauma craniectomy for refractory intracranial hypertension with severe traumatic brain injury: a multicenter, prospective, randomized controlled study. J Neurotrauma 2005;22(6): 623-628

2 Bao YH, Liang YM, Gao GY, Pan YH, Luo QZ, Jiang JY. Bilateral decompressive craniectomy for patients with malignant diffuse brain swelling after severe traumatic brain injury: a 37-case study. J Neurotrauma 2010;27(2):341-347

3 Yoo DS, Kim DS, Cho KS, Huh PW, Park CK, Kang JK. Ventricular pressure monitoring during bilateral decompression with dural expansion. J Neurosurg 1999;91(6):953-959

4 Yamaura A, Makino H. Neurological deficits in the presence of the sinking skin flap following decompressive craniectomy. Neurol Med Chir (Tokyo) 1977;17(1, Pt 1):43-53

5 Cavuşoğlu H, Kaya RA, Türkmenoğlu ON, Aydin Y. Value of early unilateral decompressive craniectomy in patients with severe traumatic brain injury. Ulus Travma Acil Cerrahi Derg 2010; 16(2):119-124

6 Münch E, Horn P, Schürer L, Piepgras A, Paul T, Schmiedek P. Management of severe traumatic brain injury by decompressive craniectomy. Neurosurgery 2000;47(2):315-322, discussion 322-323
7 Strik HM, Borchert H, Fels C, et al. Three-dimensional reconstruction and volumetry of intracranial haemorrhage and its mass effect. Neuroradiology 2005;47(6):417-424

8 Blatter DD, Bigler ED, Gale SD, et al. Quantitative volumetric analysis of brain MR: normative database spanning 5 decades of life. AJNR Am J Neuroradiol 1995;16(2):241-251

9 Whitfield PC, Patel H, Hutchinson PJ, et al. Bifrontal decompressive craniectomy in the management of posttraumatic intracranial hypertension. Br J Neurosurg 2001; 15(6):500-507

10 Cooper PR, Hagler H, Clark WK, Barnett P. Enhancement of experimental cerebral edema after decompressive craniectomy: implications for the management of severe head injuries. Neurosurgery 1979;4(4):296-300

11 Lobato RD, Sarabia R, Cordobes F, et al. Posttraumatic cerebral hemispheric swelling. Analysis of 55 cases studied with computerized tomography. J Neurosurg 1988;68(3):417-423

12 Stiver SI. Complications of decompressive craniectomy for traumatic brain injury. Neurosurg Focus 2009;26(6):E7

13 Schmidt JH III, Reyes BJ, Fischer R, Flaherty SK. Use of hinge craniotomy for cerebral decompression. Technical note. J Neurosurg 2007;107(3):678-682

14 Mitchell P, Tseng M, Mendelow AD. Decompressive craniectomy with lattice duraplasty. Acta Neurochir (Wien) 2004;146(2): 159-160

15 Csókay A, Pataki G, Nagy L, Belán K. Vascular tunnel construction in the treatment of severe brain swelling caused by trauma and $\mathrm{SAH}$. (evidence based on intra-operative blood flow measure). Neurol Res 2002;24(2):157-160

16 Alves OL, Bullock R. "Basal durotomy" to prevent massive intraoperative traumatic brain swelling. Acta Neurochir (Wien) 2003; 145(7):583-586, discussion 586

17 Wang Y, Wang C, Yang L, et al. Controlled decompression for the treatment of severe head injury: a preliminary study. Turk Neurosurg 2014;24(2):214-220

18 Olivecrona M, Rodling-Wahlström M, Naredi S, Koskinen LO. Effective ICP reduction by decompressive craniectomy in patients with severe traumatic brain injury treated by an ICP-targeted therapy. J Neurotrauma 2007;24(6):927-935 